\title{
Calculation of multidimensional potential energy surfaces for even-even transuranium nuclei: systematic investigation of the triaxiality effect on the fission barrier *
}

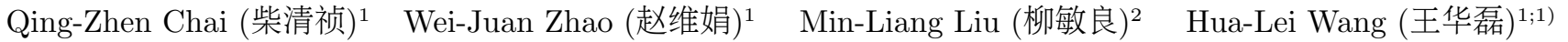 \\ ${ }^{1}$ School of Physics and Engineering, Zhengzhou University, Zhengzhou 450001, China \\ ${ }^{2}$ Institute of Modern Physics, Chinese Academy of Sciences, Lanzhou 730000, China
}

\begin{abstract}
Static fission barriers for 95 even-even transuranium nuclei with charge number $Z=94-118$ have been systematically investigated by means of pairing self-consistent Woods-Saxon-Strutinsky calculations using the potential energy surface approach in multidimensional $\left(\beta_{2}, \gamma, \beta_{4}\right)$ deformation space. Taking the heavier ${ }^{252} \mathrm{Cf}$ nucleus (with the available fission barrier from experiment) as an example, the formation of the fission barrier and the influence of macroscopic, shell and pairing correction energies on it are analyzed. The results of the present calculated $\beta_{2}$ values and barrier heights are compared with previous calculations and available experiments. The role of triaxiality in the region of the first saddle is discussed. It is found that the second fission barrier is also considerably affected by the triaxial deformation degree of freedom in some nuclei (e.g., the $Z=112-118$ isotopes). Based on the potential energy curves, general trends of the evolution of the fission barrier heights and widths as a function of the nucleon numbers are investigated. In addition, the effects of Woods-Saxon potential parameter modifications (e.g., the strength of the spin-orbit coupling and the nuclear surface diffuseness) on the fission barrier are briefly discussed.
\end{abstract}

Key words: static fission barriers, potential energy surface approach, triaxiality, Woods-Saxon potential

PACS: $21.10 . R e, 21.60 . C s, 21.60 . E v \quad$ DOI: $10.1088 / 1674-1137 / 42 / 5 / 054101$

\section{Introduction}

Transuranium nuclei are produced artificially in heavy-ion induced nuclear fusion reactions. The production rates by present production paths are allowed on an atom-at-a-minute to atom-at-a-month scale applying currently experimental techniques. There is no doubt, with the development of radioactive beam facilities, heavy-ion accelerators, and highly effective detector arrays, that there will be increasing interest in further attacking transuranium nuclei, especially the island of stability of superheavy elements [1]. Both physics and chemistry have arrived on the shore of this fascinating island in experiments [2 4]. As one of the important decay modes of a heavy or superheavy nucleus, the spontaneous fission channel is dominated essentially by the barrier size and shape [5]. Also, the formation probability of such a nucleus in heavy-ion-fusion reactions is directly related to the fission barrier, generally including its height and full-width at half-maximum (FWHM), since the barrier is a decisive quantity in the competition between neutron evaporation and fission of a compound nucleus during the process of its cooling. The production cross section of such fissioning nuclei has a sensitive dependence on the barrier shape and height. For instance, a $1 \mathrm{MeV}$ change in the fission barrier may result in a difference of several orders of magnitude in survival probability. Moreover, the fission barrier of very neutron-rich nuclei can affect the $r$-process of stellar nucleosynthesis [6, 77. Therefore, the fission barrier is a critical quantity for understanding the questions mentioned above.

Accurately describing fission been a long standing problem ever since it was deduced, for the first time, by barrier penetration about 80 years ago 8 . More recently, considerable effort has been made to understand the fission problem in both theory and experiment. Some empirical barriers in transuranium nuclei have been determined experimentally [9] and various theoretical approaches have been used for the study of the fission barriers. It has been found that different deformation degrees

Received 6 February 2018, Published online 20 April 2018*

* Supported by the National Natural Science Foundation of China (Nos. 11675148 and 11505157), the Project of Youth Backbone Teachers of Colleges and Universities of Henan Province (No. 2017GGJS008), the Foundation and Advanced Technology Research Program of Henan Province (No. 162300410222), the Outstanding Young Talent Research Fund of Zhengzhou University (No. 1521317002) and the Physics Research and Development Program of Zhengzhou University (No. 32410017).

1) E-mail: wanghualei@zzu.edu.cn

(c) 2018 Chinese Physical Society and the Institute of High Energy Physics of the Chinese Academy of Sciences and the Institute of Modern Physics of the Chinese Academy of Sciences and IOP Publishing Ltd 
of freedom can have different influence on the inner and outer barriers, though the impact may strongly depend on the proton and neutron numbers and the employed models. For instance, the heights of the inner and outer barriers can usually be lowered by the triaxiality and octupole correlation, respectively [10, 11]. Further, it was recently found that the outer barriers can also be lowered by the triaxiality compared with axially symmetric results in the actinide region [12, 13. The most fundamental way to determine the nuclear properties is to start with a real nucleon-nucleon interaction and solve the appropriate many-body equations in some approximation. However, due to the computational difficulties and complications (e.g., the hard-core property in realistic nuclear force), in practical calculation, some simple effective interactions whose parameters are adjusted to reproduce gross nuclear properties rather than nucleonnucleon scattering data, even the one-body potential, are usually used by combining a phenomenological or selfconsistent mean-field approach. Presently, there are four types of models which are widely used for investigating fission barriers, including the macroscopic-microscopic (MM) models [14 20], the nonrelativistic energy density functionals based on zero-range Skyrme and finite-range Gogny interactions 21 24, the extended Thomas-Fermi plus Strutinsky integral (ETFSI) methods [7, 25], and the covariant density functional theory (CDFT) 26 29. The difference in the description of inner fission barrier height in these models is considerable, which will translate into huge uncertainties in the spontaneous fission half-lives 30. The MM approaches with the "oldest" ages usually have very high descriptive power as well as simplicity of calculation. In this paper, the multidimensional potential energy surface (PES) calculations are based on the framework of MM models.

Experimental data show some unexpected spectroscopic characteristics in some nuclei, such as wobbling, signature inversion (or splitting) and chiral doublets 31 . 33, indicating the possible appearance of triaxiality. Prior to this work, we have performed several studies of the effects of triaxial deformation degree of freedom on ground and/or yrast state in specific isotopes such as $\mathrm{Ba}$ 34, Nd 35] and W [36] with a similar PES calculation method. Additionally, we have systematically investigated the octupole effects on outer fission barriers for even-even nuclei with $102 \leq Z \leq 112$ [37]. In this work we perform a systematic investigation of the inner fission barriers in all synthesized tranuranium nuclei within the triaxial multidimensional PES approach, focusing on the formation mechanism of the barriers and triaxiality effects, which is expected to provide a valuable reference for experiments, and to some extent even for more microscopic and relativistic calculations. The barrier shape, including its height, width and evolution, is analyzed. To our knowledge, such systematic investigations of the effects of triaxial degrees of freedom on the height of inner fission barriers are somewhat scarce for all even-even transuranium nuclides ranging from ${ }^{226} \mathrm{Pu}$ to ${ }^{294} \mathrm{Og}$ which have already been synthesized experimentally [4, 38 40]. Moreover, a systematic study may be the best way to understand the underlying principles behind the impact of triaxiality on the inner fission barriers, since it can eliminate the arbitrariness of conclusions with respect to the choice of a specific nucleus. Part of the aim of this work is to test the model validity and predictive power, especially extrapolating towards the the superheavy region, and to find discrepancies for further developing the present model. Indeed, for instance, it has been pointed out that different polarization effects and functional forms of the densities may appear in the superheavy region, which can be naturally incorporated within the self-consistent nuclear mean-field calculations. However, in the MM models, prior knowledge about the expected densities and single-particle potentials is needed 41. In general, the model parameters may need to be refitted and even the model Hamiltonian will need to be remodelled (e.g., see Refs. 42 44]).

The rest of this paper is organized as follows. The theoretical framework and the details of the numerical calculations are described in Section 2. The calculated fission barriers, the effect of triaxiality, and the comparison with data and other theoretical results are presented in Section 3. Finally, Section 4 summarizes the main conclusions of the present work.

\section{Theoretical framework}

The PES calculation [45] employing in the present work is based on the MM models [46, 47, which are approximations of the self-consistent Hartree-Fock method [48, 49]. Such approaches have been widely used to reproduce the right bulk properties, e.g., the groundstate deformations and energies, of a many-body system in medium and heavy mass nuclei [50, 51]. In this section, the common procedures are outlined, with some helpful references.

The basic idea in the MM models is that the total potential energy of a deformed nucleus can be decomposed into two parts,

$$
E_{\text {total }}(Z, N, \hat{\beta})=E_{\mathrm{mac}}(Z, N, \hat{\beta})+E_{\mathrm{mic}}(Z, N, \hat{\beta}),
$$

where $E_{\text {mac }}$ is the macroscopic energy given by a smooth function of nucleon numbers $(Z, N)$ and deformations $(\hat{\beta})$, while $E_{\text {mic }}$ represents the microscopic quantum correction calculated from a phenomenological (non-selfconsistent) single-particle potential well. Generally, the unified procedure of such an approach is then carried out in the following five steps [46]: 
(a) Specify the nuclear shape, i.e., shape parameterization.

(b) Calculate the macroscopic energy, e.g., liquiddrop (LD) energy.

(c) Generate the single-particle potential felt by protons or neutrons, e.g., the Nilsson, Woods-Saxon (WS), and folded Yukawa (FY) potentials.

(d) Solve the stationary Schrödinger equation to obtain the single-particle levels and wave functions.

(e) Calculate microscopic (shell and pairing) corrections.

The total potential energy can obviously be obtained by the sum of macroscopic and microscopic energies given in steps (b) and (e), respectively. Next, each part will be introduced according to these five steps.

First of all, the nuclear shape can be conveniently described by the parametrization of the nuclear surface or the nucleon density distribution. In the present work, the nuclear surface $\Sigma$ is depicted with the multipole expansion of spherical harmonics $Y_{\lambda \mu}(\theta, \phi)$, that is,

$$
\Sigma: R(\theta, \phi)=R_{0}\left[1+\sum_{\lambda} \sum_{\mu=-\lambda}^{+\lambda} \alpha_{\lambda \mu} Y_{\lambda \mu}^{*}(\theta, \phi)\right]
$$

where $R_{0}$ is the radius of the isovolume spherical shape, which is determined by requiring conservation of the nuclear volume equal to $4 \pi R_{0}^{3} / 3$, and $\hat{\beta}$ stands for all the deformation parameters applied here. Note that such parametrization is also convenient to describe the nuclear geometrical symmetry. Here, the dominating loworder quadrupole deformations $\left(\alpha_{20}, \alpha_{2 \pm 2}\right)$ and hexadecapole deformations $\left(\alpha_{40}, \alpha_{4 \pm 2}, \alpha_{4 \pm 4}\right)$ have been included. Meanwhile, the nuclear surface radius $R(\theta, \phi)$ represents the distance of a point between the nuclear surface and the origin of the corresponding coordinate system. Since only the even $\lambda$ and $\mu$ components are included, the nuclear shape will obviously survive three symmetry planes by such parametrization. Furthermore, once the hexadecapole deformation is taken into account with the functions of the scalars in the quadrupole tensor $\alpha_{2 \mu}$, it can lead to a three-dimensional calculation with the independent coefficients $\beta_{2}, \gamma$ and $\beta_{4}$ [52, i.e.,

$$
\left\{\begin{array}{l}
\alpha_{20}=\beta_{2} \cos \gamma \\
\alpha_{22}=\alpha_{2-2}=\frac{\sqrt{2}}{2} \beta_{2} \sin \gamma \\
\alpha_{40}=\frac{1}{6} \beta_{4}\left(5 \cos ^{2} \gamma+1\right) \\
\alpha_{42}=\alpha_{4-2}=-\frac{1}{12} \sqrt{30} \beta_{4} \sin 2 \gamma \\
\alpha_{44}=\alpha_{4-4}=\frac{1}{12} \sqrt{70} \beta_{4} \sin ^{2} \gamma .
\end{array}\right.
$$

Of course, the $\left(\beta_{2}, \gamma, \beta_{4}\right)$ parametrization has all the symmetry properties (e.g., axial symmetry and reflection symmetry) of Bohr's $\left(\beta_{2}, \gamma\right)$ parametrization [53, 54].

Secondly, up to now, there are several phenomenological LD models which have been used in the litera- ture, e.g., standard LD model [55], finite-range liquiddrop model (FRLDM) [56, finite-range droplet model (FRDM) [57, Lublin-Strasbourg drop (LSD) model [58], etc. These macroscopic models with slightly different properties can be utilized to give the smoothly varying part of the nuclear energy, in which the dominating terms involve the volume energy, the surface energy and the Coulomb energy. Due to the incompressibility of a nucleus ("volume conservation" condition), the volume energy, which is proportional to mass number $A$, does not depend on the nuclear shape, whereas the surface energy, which tends to hold the nucleus together, and the Coulomb energy, which tends to pull the nucleus apart, are shape-dependent. Here, the macroscopic energy calculated in the present work is obtained by the standard LD model with the parameter set used by Myers and Swiatecki [55]. Because we are focusing on the PES and the difference between the critical points (e.g., between the minimum and saddle point), the potential energy relative to the energy of a spherical LD has been adopted. This portion of the standard LD energy can be can be written as [55, 59, 60.

$$
E_{\mathrm{LD}}(Z, N, \hat{\beta})=\left\{\left[B_{s}(\hat{\beta})-1\right]+2 \chi\left[B_{c}(\hat{\beta})-1\right]\right\} E_{s}^{(0)},
$$

where the relative surface energy $B_{s}$ and Coulomb energy $B_{c}$ are functions only of nuclear shape, depending on the collective coordinates $\left\{\alpha_{\lambda \mu}\right\}$. The spherical surface energy $E_{s}^{(0)}$ and the fissility parameter $\chi$ are $Z$ - and $N$-dependent. The detailed expression of $E_{s}^{(0)}$ and $\chi$ can be found in Ref. [60].

Thirdly and fourthly, in the one-body mean-field approximation, for example, the Nilsson (modified harmonic-oscillator), WS and FY potentials are usually adopted during the process of real calculations [46, 61, 62. At present, we use a more realistic diffuse-surface deformed WS-type nuclear potential. That is, the singleparticle levels and wave functions are determined by solving the stationary Schrödinger equation numerically with $H_{\mathrm{wS}}$ [63],

$$
\begin{aligned}
H_{\mathrm{WS}}= & -\frac{\hbar^{2}}{2 m} \nabla^{2}+V_{\text {cent }}(\vec{r} ; \hat{\beta})+V_{\mathrm{so}}(\vec{r}, \vec{p}, \vec{s} ; \hat{\beta}) \\
& +\frac{1}{2}\left(1+\tau_{3}\right) V_{\mathrm{Coul}}(\vec{r}, \hat{\beta}),
\end{aligned}
$$

where the depth of the central part of the WS potential, which will mainly govern the number of levels in the potential well, is [59],

$$
V=V_{0}[1 \pm \kappa(N-Z) /(N+Z)],
$$

with the plus and minus signs for protons and neutrons respectively, and the values of the constants $V_{0}$ and $\kappa$ given later. Then the spin-orbit potential $V_{\text {so }}(\vec{r}, \vec{p}, \vec{s} ; \hat{\beta})$, which mainly controls the relative positions of levels, is 
assumed of the form

$$
\begin{aligned}
V_{\mathrm{so}}(\vec{r}, \vec{p}, \vec{s} ; \hat{\beta})= & -\lambda\left[\frac{\hbar}{2 m c}\right]^{2} \\
& \left\{\nabla \frac{V_{0}[1 \pm \kappa(N-Z) /(N+Z)]}{1+\exp \left[\operatorname{dist}_{\Sigma_{\mathrm{so}}}(\vec{r}, \hat{\beta}) / a_{\mathrm{so}}\right]}\right\} \times \vec{p} \cdot \vec{s},
\end{aligned}
$$

where $\lambda$ is the strength parameter of the spin-orbit potential and the new surface $\Sigma_{\text {so }}$ denotes the surface of the spin-orbit potential. In addition, the Coulomb potential $V_{\text {Coul }}(\vec{r}, \hat{\beta})$ for protons is considered as a uniformly charged drop in a classical electrostatic potential.

The universal WS parameter set [59] has been applied in the present work. This parameter set is $Z$ and $N$ independent and can give a reliable description of the singleparticle states, especially in the medium and heavy mass regions [64. As shown in Refs. 47, 59, these parameters are:

(1) Central potential depth parameters: $V_{0}=49.6 \mathrm{MeV}, \kappa=0.86$.

(2) Radius parameters of the central part: $r_{0}(p)=1.275 \mathrm{fm}, r_{0}(n)=1.347 \mathrm{fm}$.

(3) Radius parameters of the spin-orbit part: $r_{0-\mathrm{so}}(p)=1.320 \mathrm{fm}, r_{0-\mathrm{so}}(n)=1.310 \mathrm{fm}$.

(4) Strength of the spin-orbit potential: $\lambda(p)=36.0, \lambda(n)=35.0$.

(5) Diffuseness parameters: $a_{0}(p)=a_{0}(n)=a_{0-\mathrm{so}}(p)=a_{0-\mathrm{so}}(n)=0.70 \mathrm{fm}$.

These are usually fitted by adjusting their values to optimally reproduce the energies of available single-particle levels in either spherical or deformed nuclei 65 68. It is worth noting that these parameters may be not constant throughout the global nuclear chart and need to be adjusted somehow when extrapolating to the unknown region. Based on the above parameters, the WS singleparticle potential felt by protons and neutrons is generated clearly at the deformation space $\left(\beta_{2}, \gamma, \beta_{4}\right)$. Then the WS Hamiltonian matrix is calculated using the basis of the axially deformed harmonic oscillator in the cylindrical coordinate system. Finally, the single-particle levels and wave functions can be obtained by diagonalizing the Hamiltonian matrix. Note that the harmonic oscillator eigenfunctions with the principal quantum number $N \leq 12$ and $N \leq 14$ are adopted during the calculation as a basis for protons and neutrons respectively, since such a basis cutoff can give stable results in the case of its possible enlargement.

Fifthly, the microscopic energy arises as a result of the inhomogeneous distribution of the single-particle energies and the residual pairing interaction in the nucleus, mainly consisting of a shell correction $\delta E_{\text {shell }}$ and a pairing correction $\delta E_{\text {pair }}$, namely,

$$
E_{\text {mic }}(Z, N, \hat{\beta})=\delta E_{\text {shell }}(Z, N, \hat{\beta})+\delta E_{\text {pair }}(Z, N, \hat{\beta}) .
$$

Based on the single-particle levels obtained above, the shell and pairing corrections at each deformation point $\left(\beta_{2}, \gamma, \beta_{4}\right)$ can be evaluated by means of the Strutinsky method [48] and Lipkin-Nogami (LN) method 69]. The Strutinsky smoothly varying part is carried out with a six-order Laguerre polynomial with the smoothing range $\gamma=1.20 \hbar \omega_{0}\left(\hbar \omega_{0}=41 / A^{1 / 3} \mathrm{MeV}\right)$. The LN method, in which particle number projection is approximately conserved, avoids the spurious pairing phase transition encountered in the traditional Bardeen-Cooper-Schrieffer (BCS) calculation. The monopole pairing has been considered and its strength, $G$, is obtained from the average gap method [70]. In the pairing windows, the respective states, e.g., half of the particle number $Z$ and $N$ (or 40 , if they are greater than 40) just below and above the Fermi energy, are included empirically for both protons and neutrons. Then the energy in the LN approach is given by [62, 69],

$E_{\mathrm{LN}}=\sum_{k} 2 v_{k}^{2} e_{k}-\frac{\Delta^{2}}{G}-G \sum_{k} v_{k}^{4}+G \frac{N}{2}-4 \lambda_{2} \sum_{k} u_{k}^{2} v_{k}^{2}$,

where $v_{k}^{2}, e_{k}$ and $\Delta$ denote the occupation probabilities, single-particle energies and pairing gap respectively. The extra Lagrange multiplier $\lambda_{2}$ represents the particle-number-fluctuation constant. Further, the shell and pairing corrections could be calculated by $\delta E_{\text {shell }}=$ $\sum e_{i}-\tilde{E}_{\mathrm{Strut}}$ and $\delta E_{\mathrm{pair}}=E_{\mathrm{LN}}-\sum e_{i}$, respectively. Here, $\sum e_{i}$ is the sum of single-particle energies and $\tilde{E}_{\mathrm{Strut}}$ is the smoothing energy by the Strutinsky method.

Last but not least, taking both Bohr shape deformation parameters [53] and the Lund convention [71] into account, the Cartesian quadrupole coordinates $X=$ $\beta_{2} \cos \left(\gamma+30^{\circ}\right)$ and $Y=\beta_{2} \sin \left(\gamma+30^{\circ}\right)$ are used in the present work, where $\beta_{2}$ specifies the magnitude of the quadrupole deformation and $\gamma$ describes nonaxial shapes. In these calculations, the $\beta_{2}$ value "built-in" is always positive and the $\gamma$ value covers the range $-120^{\circ} \leq$ $\gamma \leq 60^{\circ}$. Obviously, the three sectors $\left[-120^{\circ},-60^{\circ}\right]$, $\left[-60^{\circ}, 0^{\circ}\right]$ and $\left[0^{\circ}, 60^{\circ}\right]$ describe the identical triaxial shapes at the ground state. At each $(X, Y)$ deformation grid, the total energy of a nucleus is calculated according to the procedure mentioned above and the PES can finally be derived from interpolating, using a cubic spline function, between the lattice points in the $(X, Y)$ plane. Therefore, the nuclear properties such as the ground-state equilibrium deformations, saddle points, fission paths and so on can be obtained and analyzed based on the present PES calculation.

\section{Results and discussion}



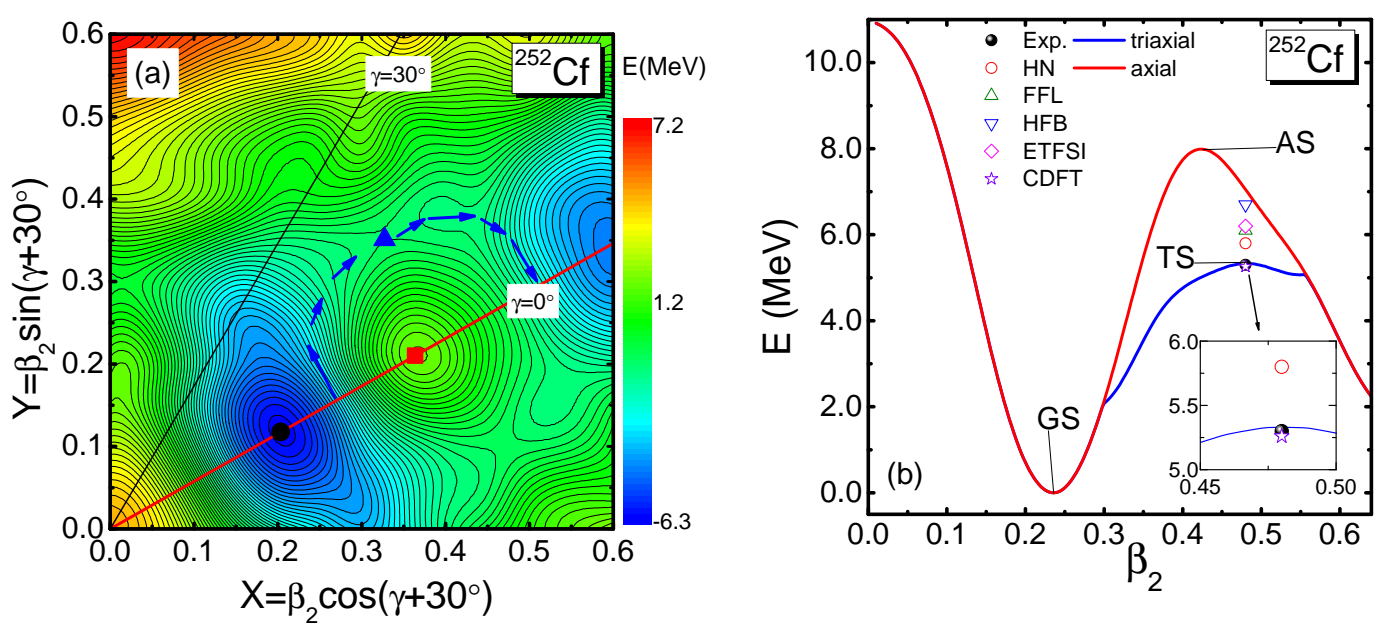

Fig. 1. (a) Calculated two-dimensional PES in $\left(\beta_{2}, \gamma, \beta_{4}\right)$ deformation space for the selected nucleus ${ }^{252} \mathrm{Cf}$. At each $\left(\beta_{2}, \gamma\right)$ grid point, the PES has been minimized with respect to the deformation parameter $\beta_{4}$. The PES minimum, namely, the ground state (GS), is indicated by the black circle. The red square and blue triangle represent the axial saddle (AS) and triaxial saddle (TS) along the axial (red line) and triaxial (blue line) fission paths, respectively. The energy contours are at $200 \mathrm{keV}$ intervals. (b) Calculated potential energy curves against $\beta_{2}$ for ${ }^{252} \mathrm{Cf}$. At each given $\beta_{2}$ point, the energy has been minimized with respect to $\gamma$ and $\beta_{4}$ deformations. Similar to (a), the red solid line displays the potential energy curve for the axially symmetric solution with $\gamma=0^{\circ}$, whereas the blue solid line shows the corresponding curve along the triaxial part of the fission trajectory which has the lowest energy as a function of $\beta_{2}$. For convenience of description, the energy curve is normalized with respect to the ground-state energy. The experimental and other several theoretical fission barriers are shown for comparison. Further details are given in the text.

In the present work, we just care about the inner fission barriers rather than the outer ones. Such a restriction has its own merits. The inner barriers are easier to measure than the outer barriers, and they are more important for the $r$ process since they determine thresholds. In addition, spontaneous fission lifetimes tend to be dominated by the inner barrier, even if an outer barrier can occasionally have a crucial influence if it is wide enough. Previous studies [15, 24, 26, 72,74, have shown that the odd-multipole deformations (e.g., $\beta_{3}$ ) do not play a role in the inner fission barrier of the actinide and superheavy regions. This allows us to restrict our calculations to reflection symmetric shapes. It has also been shown that the inclusion of triaxiality can improve the accuracy of the description of the inner fission barriers in the actinide region in almost all state-of-the-art models [13, 16, 17, 29, 75]. Therefore, taking the important low-order deformation degrees of freedom into account, we performed the PES calculation in multidimensional $\left(\beta_{2}, \gamma, \beta_{4}\right)$ space, paying attention to the impact of triaxiality on the inner fission barrier, especially in the superheavy nuclei. To examine the model validity in the current research, as an example, the ${ }^{252} \mathrm{Cf}$ nucleus is calculated, as it is the closest to the superheavy region among the nuclei that have experimentally measured inner fission barriers [9, 76. Figure 1 shows the calculated 2D PES (a) and the triaxial and axial 1D po- tential energy curves (b) for ${ }^{252} \mathrm{Cf}$. The axial and triaxial fission paths, saddles and equilibrium deformations can be clearly seen in Fig. 1(a). For instance, one can see that this nucleus has a well-deformed prolate shape and a triaxial-deformed saddle, $\gamma \sim 17^{\circ}$, which will strongly modify the fission path. The "real" fission path will go through the energy valley (denoted by the blue line) with a non-zero $\gamma$ deformation in the $\left(\beta_{2}, \gamma\right)$ plane by avoiding the maximum of the axially symmetric PES. The properties of the inner fission barrier, including its height and width, are shown in Fig. 1.(b). The triaxiality lowers the barrier height of this nucleus by up to $2.66 \mathrm{MeV}$ here. For comparison, the experimental data and other several theoretical results of the inner fission barrier for ${ }^{252} \mathrm{Cf}$ are also shown. Note that these several theoretical results are, respectively, obtained from the so-called heavy nuclei (HN) model [19], the FY single-particle potential and the FRLDM [17, the Skyrme-Hartree-Fock-Bogoliubov (SHFB) method [74, the ETFSI methods 7] and the CDFT theory [13. It can be seen that all the calculated values are higher than the data except for the CDFT value, which has a $40 \mathrm{keV}$ underestimation. Interestingly, our result shows good agreement with experimental data, at least in this nucleus, with only a $\sim 30 \mathrm{keV}$ difference.

Nowadays, it is well understood that the minima (ground or shape-coexisting states) and maxima (or sad- 
dle points) can be attributed to shell effects, whose microscopic mechanism originates from the nonuniform distribution of the single-particle levels in the vicinity of the Fermi surface [4]. Minima correspond to regions of low level density, e.g., a region with a large energy gap, whereas saddle points usually occur in the vicinity of level crossings, regions of high level density. The fission barrier (namely, the energy difference between the ground-state minimum and the corresponding saddle point) is certainly related to the level density near the Fermi level. For additional clarity and emphasis, Fig. 2 shows calculated proton and neutron single-particle levels near the Fermi surface at three typical deformation points (ground-state minimum, axial and triaxial saddles) for the selected ${ }^{252} \mathrm{Cf}$ nucleus. The single-particle level density near the Fermi level at the ground-state minimum is lower than those at the saddles, indicating a large negative shell correction energy. Especially, it is very clear that the highest neutron level density is near the Fermi level at the axial saddle point, as seen in Fig. 2(b), since at both the ground-state minimum and triaxial saddle the levels are shifted up above the Fermi level and down below the Fermi level (leading to a relatively lower level density). The decrease of the triaxial saddle (or the lowering of the barrier due to triaxiality) can certainly be understood by such a level density change.

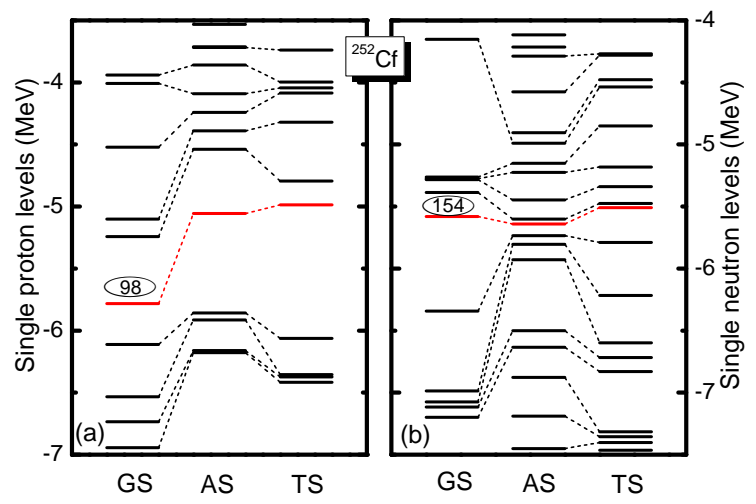

Fig. 2. Calculated proton (a) and neutron (b) single-particle levels near the Fermi surface for ${ }^{252} \mathrm{Cf}$ at three typical deformation grid points, that is, GS, AS and TS points, as seen in Fig. 1 The red lines indicate the Fermi energy levels.

To give a better understanding of the macroscopic and microscopic contributions in the calculated PES, especially at critical minima and saddle points, Fig. 3 shows the calculated potential energy curves and corresponding histograms for ${ }^{252} \mathrm{Cf}$. It can be seen from Fig. 3(a) that the inclusion of triaxiality does not change the first minimum but strongly affects the shape and height of the first barrier. The macroscopic energy changes smoothly and the triaxiality will lead to an additional energy increase. However, the microscopic energy fluctuates significantly and can be strongly affected by the triaxiality, mostly determining the positions of the minima and saddles. Note that at the zero deformation point, the reason for $E_{\text {macro }}=0$ is that the macroscopic energy is normalized to the spherical liquid drop, as mentioned in the theoretical part. More intuitively, Fig. 3(b) shows us to what extent the energy of the minimum and saddles are affected by the macroscopic energy and microscopic correction.
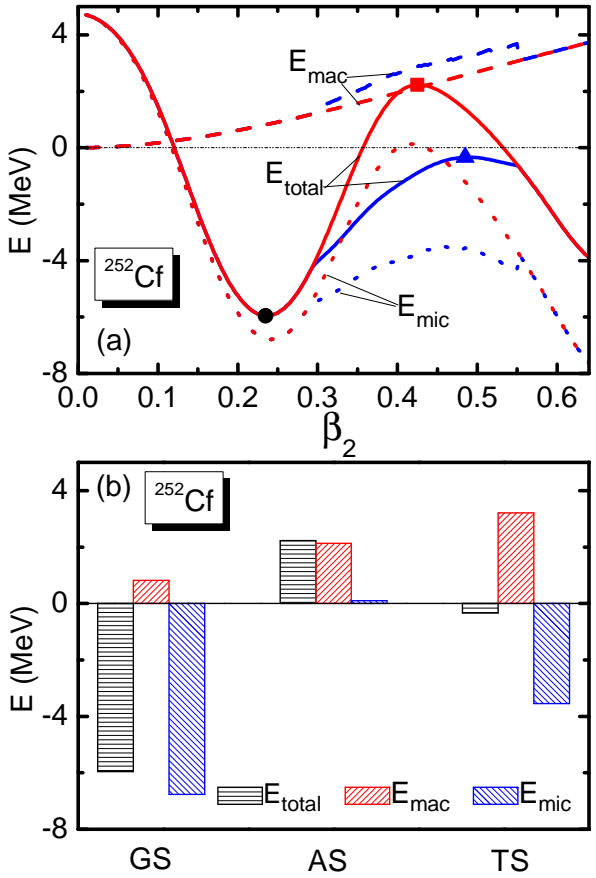

Fig. 3. (a) Similar to Fig. 1(b), calculated potential energy curves against $\beta_{2}$ for ${ }^{252} \mathrm{Cf}$, but together with the macroscopic and microscopic components. The circle, triangle and square symbols represent the ground state (GS), axial saddle (AS) and triaxial saddle (TS), respectively. Note that, for simplicity, the $\beta_{4}$ deformation is not considered here. (b) The calculated total energy and its macroscopic and microscopic contributions at the GS, AS and TS points of part (a).

Aside from the mean field, which determines the single-particle levels, the pairing correlations can also affect the calculated barrier to some extent. For example, it has been pointed out that there may be a rather large difference in the predicted barrier heights between different pairing models [26, 29, 77]. In addition, with the dynamical coupling between shape and pairing degrees of freedom, the fission barrier also will be reduced by several units of least-action of fission paths [78. Here, to obtain a crude estimation of the contribution due to the pairing correlation, as shown in Fig. 4. the microscopic energy correction has been further divided into shell and pairing corrections. One can see that the $\beta_{4}$ deformation can slightly modify the different energy components. As 
expected, it is found that the shell corrections are fully in agreement with the cases of the density distribution, as seen in Fig. 2, For instance, the ground-state minimum has the lowest level density, corresponding to the largest negative shell correction and the smallest negative pairing correction, whereas the axial saddle point has the highest level density, corresponding to a positive shell correction and the largest negative pairing correction. It seems that the triaxiality will change the single-particle levels by changing the phenomenological mean field, then change the shell and pairing corrections and finally have an effect on the fission barrier.

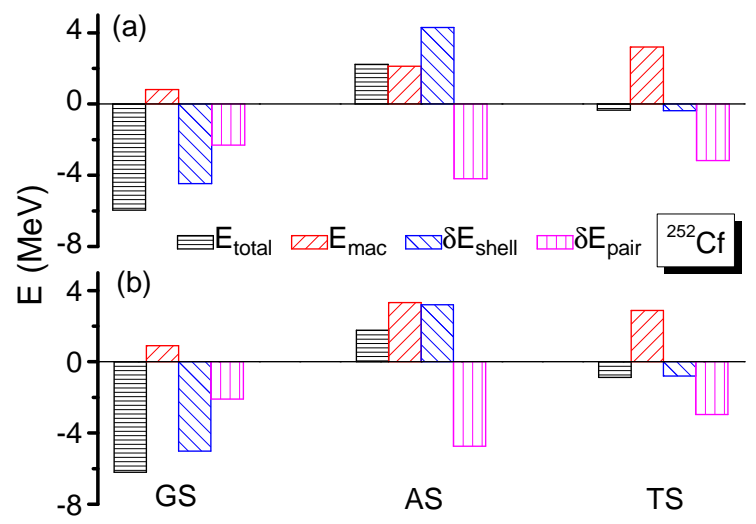

Fig. 4. (a) Similar to Fig. 3(b), but the microscopic energy is further divided into shell and pairing corrections. (b) The same as in (a), but the $\beta_{4}$ deformation is included in the calculation.
As a basic rule of scientific research, theoretical quantities generally need to be confronted with the corresponding experimental data and/or other accepted theories. Table 1 shows such two comparable quantities, the calculated ground-state equilibrium deformation parameter $\beta_{2}$ and inner fission barriers $B_{f}$, for 13 even-even transuranium nuclei where the inner fission barriers have been determined experimentally. The experimental $\beta_{2}$ values are deduced from the intrinsic quadrupole moment related to the reduced electric quadrupole transition probability $B(E 2)[79$. The other theoretical values are, respectively, obtained from the so-called $\mathrm{HN}$ model [80], the FY single-particle potential and the FRDM [81, the Hartree-Fock-BCS (HFBCS) 82, and the ETFSI methods 83 . For the inner fission barriers, the experimental or empirical values are taken from Refs. [9, 76] and the other theoretical values come from the FY single-particle potential and the FRLDM [17, the SHFB method [74] and the CDFT [13] in addition to the above mentioned HN 19 and ETFSI 7] methods. It is clearly seen that all calculated $\beta_{2}$ values are lower than the experimental results. The barriers calculated by different theories are unevenly distributed on both sides of the experimental values. None of the theories can completely reproduce the experimental data or even, as seen in the $B_{f}$ column, always come close to the experimental data. It is hard to absolutely say which one is the best, in particular when extending to unknown nuclei. However, it seems that these theories can to a large extent reproduce the deformed shapes and barrier amplitudes and support one another.

Table 1. The calculated results (PES) for ground-state equilibrium deformation parameter $\beta_{2}$ and inner fission barriers $B_{f}$ for 13 available even-even actinide nuclei. The $\beta_{2}$ values of the HN [80, FY+FRDM (FFD) 81], HFBCS 82, and ETFSI 83] calculations and experiments (Exp.) [79, and the $B_{f}$ inner fission barriers of the HN [1], FY+FRLDM (FFL) [17, SHFB [74, ETFSI [7, CDFT [1] calculations and experiments (Exp.) 9] 76] are given for comparison.

\begin{tabular}{|c|c|c|c|c|c|c|c|c|c|c|c|c|c|}
\hline \multirow[t]{2}{*}{ Nuclei } & \multicolumn{6}{|c|}{$\beta_{2}$} & \multicolumn{7}{|c|}{$B_{f}(\mathrm{MeV})$} \\
\hline & PES & $\mathrm{HN}$ & FFD & HFBCS & ETFSI & $\operatorname{Exp}^{a}$ & PES & $\mathrm{HN}$ & FFL & SHFB & ETFSI & CDFT & Exp. \\
\hline${ }^{236} \mathrm{Pu}$ & 0.215 & 0.215 & 0.215 & 0.26 & 0.22 & - & $5.72^{b}$ & 5.4 & 4.5 & 4.7 & 4.8 & - & 5.70 \\
\hline${ }^{238} \mathrm{Pu}$ & 0.220 & 0.223 & 0.226 & 0.24 & 0.24 & 0.282 & 6.32 & 6.1 & 5.3 & 5.4 & 5.4 & 5.96 & 5.60 \\
\hline${ }^{240} \mathrm{Pu}$ & 0.225 & 0.231 & 0.237 & 0.25 & 0.24 & 0.290 & 6.48 & 6.4 & 6.0 & 5.9 & 5.8 & 5.92 & 6.05 \\
\hline${ }^{242} \mathrm{Pu}$ & 0.227 & 0.233 & 0.237 & 0.24 & 0.26 & 0.298 & 6.38 & 6.3 & 6.4 & 6.3 & 6.2 & 5.77 & 5.85 \\
\hline${ }^{244} \mathrm{Pu}$ & 0.229 & 0.235 & 0.237 & 0.23 & 0.26 & 0.292 & 6.16 & 6.0 & 6.6 & 6.5 & 6.4 & 5.40 & 5.70 \\
\hline${ }^{246} \mathrm{Pu}$ & 0.233 & 0.239 & 0.250 & 0.25 & 0.26 & - & 5.76 & 5.7 & 6.3 & 6.5 & 6.2 & 4.76 & 5.40 \\
\hline${ }^{244} \mathrm{Cm}$ & 0.231 & 0.237 & 0.249 & 0.25 & 0.26 & 0.296 & 6.49 & 6.6 & 6.9 & 6.4 & 6.4 & 6.34 & 6.18 \\
\hline${ }^{246} \mathrm{Cm}$ & 0.233 & 0.240 & 0.249 & 0.27 & 0.26 & 0.298 & 6.29 & 6.2 & 7.0 & 6.7 & 6.5 & 5.84 & 6.00 \\
\hline${ }^{248} \mathrm{Cm}$ & 0.236 & 0.242 & 0.250 & 0.28 & 0.26 & 0.286 & 5.90 & 5.9 & 6.8 & 6.7 & 6.5 & 5.35 & 5.80 \\
\hline${ }^{250} \mathrm{Cm}$ & 0.234 & 0.242 & 0.250 & 0.24 & 0.26 & - & 5.39 & 5.3 & 5.9 & 6.5 & 6.5 & 4.79 & 5.40 \\
\hline${ }^{250} \mathrm{Cf}$ & 0.240 & 0.246 & 0.250 & 0.28 & 0.26 & 0.298 & 5.85 & 6.5 & 7.1 & 6.8 & 6.7 & 5.70 & 5.60 \\
\hline${ }^{252} \mathrm{Cf}$ & 0.237 & 0.246 & 0.251 & 0.25 & 0.26 & 0.304 & 5.33 & 5.8 & 6.1 & 6.7 & 6.2 & 5.26 & 5.30 \\
\hline
\end{tabular}

${ }^{a}$ The uncertainties are less than 0.015; see Ref. [79] for details.

$b$ The bold italic denotes that this fission barrier, among these theoretical values, is relatively close to experimental data. 


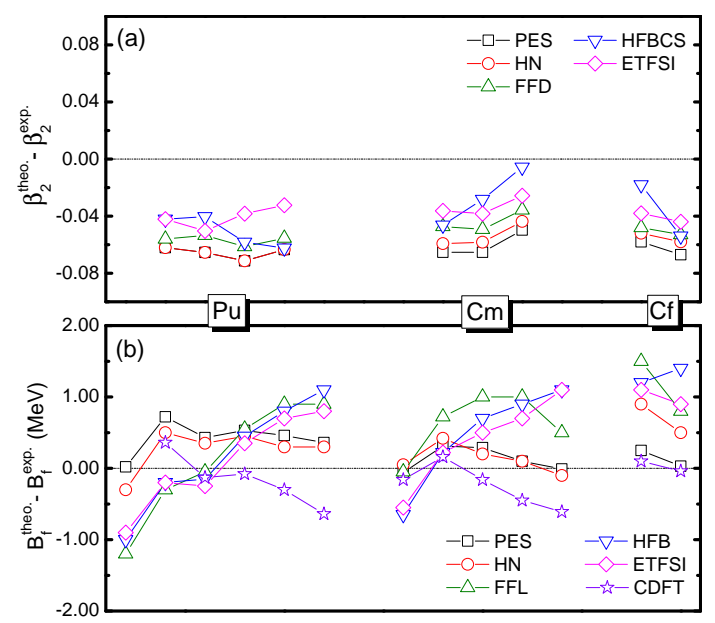

Fig. 5. (a) The difference of quadrople deformation $\beta_{2}$ between theory and available data for transuranium $\mathrm{Pu}, \mathrm{Cm}$ and $\mathrm{Cf}$ nuclei. (b) Similar to (a) but for the difference of inner fission barrier $B_{f}$.

Before continuing the investigation, we would like to further evaluate the different theoretical results by comparing the differences between calculated and experimental data. Figure 5(a) and (b) show the differences between calculated and experimental quadrople deformations $\beta_{2}$ and inner fission barriers $B_{f}$, respectively. It is seen that the calculated $\beta_{2}$ values are systematically underestimated, especially in the present work. Concerning this discrepancy, a corrected formula, e.g., for protons in the WS case, $\beta_{2}^{\rho} \simeq 1.10 \beta_{2}-0.03\left(\beta_{2}\right)^{3}$, has been suggested by Dudek et al. 84 by analyzing the relationship between the WS potential parameters and the nucleonic distributions. It is also pointed out that the vibration effect, e.g., the zero-point motion, which would imply that the experiment-comparable deformations are not static values but rather are the most likely deformations calculated from the solutions of the collective motion, may be partly responsible for such shape inconsistency [85. From Fig. 5(b), one can see that in most nuclei theoretical calculations overestimate the fission barriers, especially in $\mathrm{Cm}$ and $\mathrm{Cf}$ isotopes. It can be seen that the present PES calculation displays a relatively high descriptive power. The rms (average) deviations of the present PES, HN, FFL, HFB, ETFSI and CDFT results are $0.38(0.26), 0.44$ (0.28), 0.87 (0.48), 0.87 (0.45), $0.72(0.34)$ and $0.32(-0.16) \mathrm{MeV}$, respectively. It seems that apart from the CDFT result, our calculation gives the best description of experimental data. However, the
CDFT result is somewhat far from the other theoretical calculations, and the very small average derivation may originate from the fluctuation of the calculated values above and below the zero line (the distribution of our results is mostly above zero). In addition, our calculation gives a similar trend to the HN calculation but has smaller rms and average deviations. All these facts make us confident of the validity of our approach and of our investigation of the impact of triaxiality on the fission barrier.

Taking the above facts into account, systematic multidimensional PES calculations for 95 transuranium nuclei (including 49 actinide and 46 superheavy members) have been carried out, focusing on the influence of triaxiality on the inner fission barrier. Figures 6 and 7 show the calculated potential energy curves with and without the triaxial deformation degree of freedom for these nuclei. Note that the selected 95 nuclei ranging from $\mathrm{Pu}(Z=94)$ to $\mathrm{Og}(Z=118)$ isotopes, as seen in Figs. 6 and 7, have already been synthesized experimentally [4, 38, 40. From these two figures, it is found that the ground states of these nuclei are deformed and one can see the properties of the inner fission barriers including their heights, widths and the evolution with various nucleon numbers. Obviously, for the actinide nuclei triaxiality has a considerable impact on the inner fission barriers and makes the calculated barrier heights agree reasonably with available data. From the lower left to the upper right corner of Fig. 6, the triaxiality decreasing the fission barrier widths gradually changes to reducing the heights. The fission path has been strongly modified by the triaxiality, which will lead to a significant reduction of the penetration probability 86. In the superheavy region, as shown in Fig. 7, one can clearly see that the situation is more complicated than in the case of the actinides. The barrier shape and the effect of triaxiality in the lighter $\mathrm{Rf}$ and $\mathrm{Sg}$ isotopes are similar to those in the heavier actinide nuclei. However, in other nuclei the shapes of the fission barriers are rather different. For instance, from the "south-west" to the "north-east" direction, the fission barrier becomes wider and wider until it collapses in the intermediate section. Correspondingly, the energy curve, especially the triaxial one, becomes softer and softer (i.e., there is no easily recognizable saddle point in the heavier Rf and Sg isotopes, Hs and Ds isotopes) until the bimodal barriers appear, e.g., in the FI, Lv and Og isotopes. The bimodal barriers which appear are strongly affected by the triaxiality, as seen in Fig. 7 . 


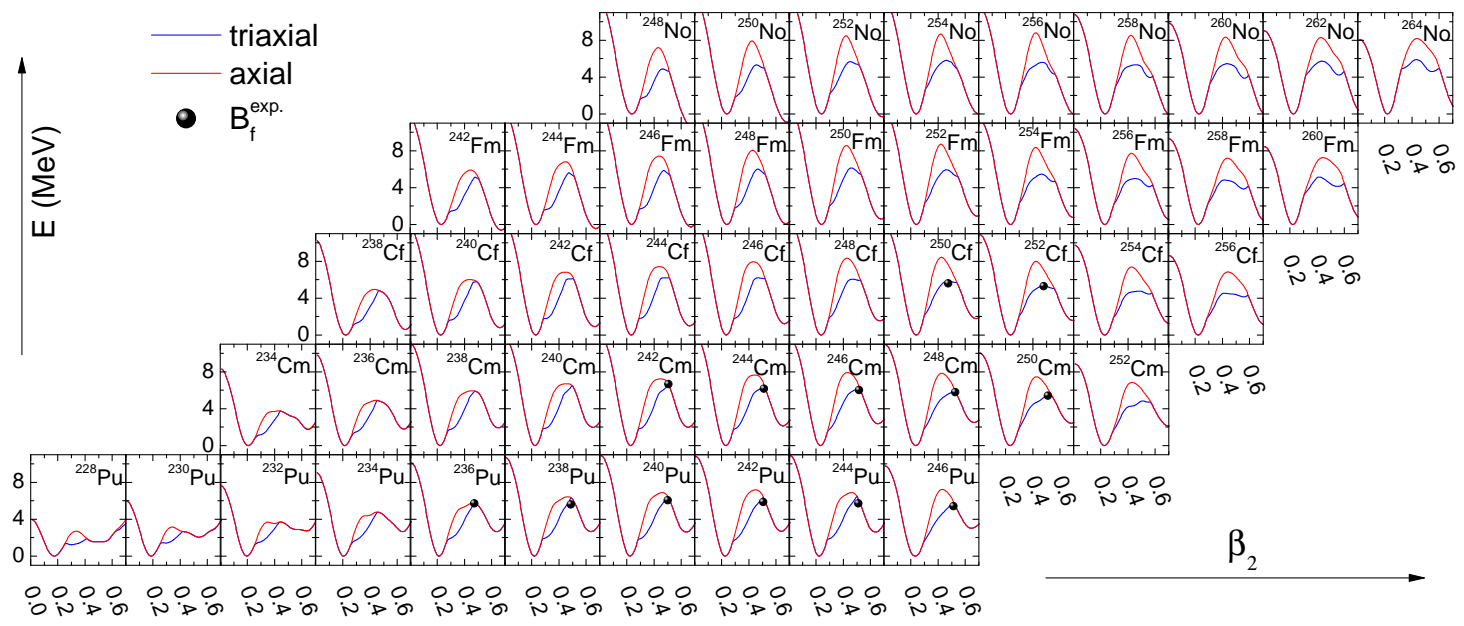

Fig. 6. Similar to Fig. 1(b), calculated potential energy curves of 49 even-even actinide nuclei with $94 \leq Z \leq 102$. The solid black circles show the available data for the height of the inner fission barrier [9, 76, as seen in Table 1 ]

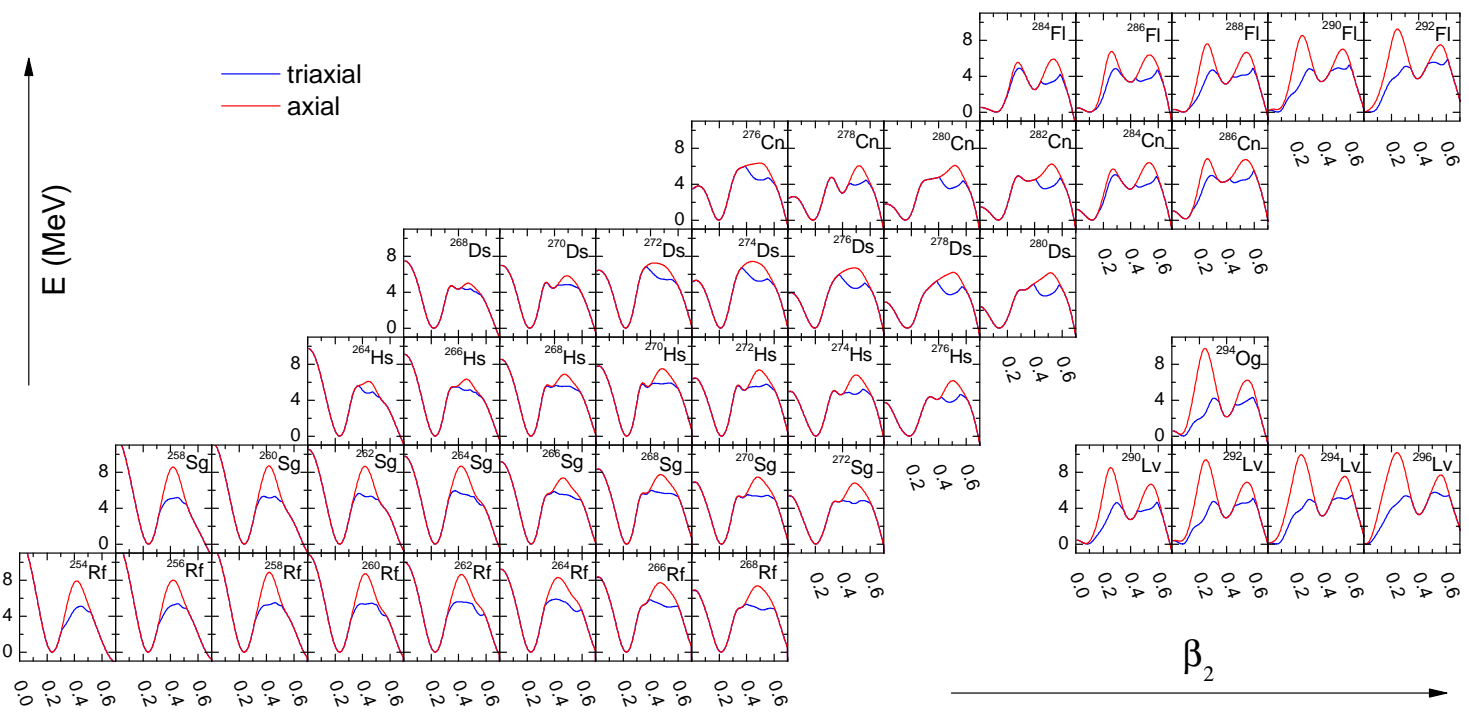

Fig. 7. The same as in Fig. 6 but for 46 even-even superheavy nuclei with $104 \leq Z \leq 118$.

In addition, in the MM calculation, besides a relatively large and reasonable deformation space, the isospin dependence of the spin-orbit coupling strength and the nuclear surface diffuseness parameter has been found to be an important factor in the accurate description of nuclear ground-state properties, especially for the extreme isospin nuclei 87, 88. Similarly, aside from the fact that the inclusion of some critical deformation degrees of freedom may greatly decrease the fission barrier, it was shown in our previous work that the adjustment of the potential parameters (e.g., the strength of the spinorbit potential, $\lambda$, and the nuclear surface diffuseness, $a$ ) can also affect the height of the fission barrier 34]. In the present transuranium region (in particular the superheavy region), it is certainly of interest to examine to what extent the fission barrier will be affected by a similar adjustment of the model parameters. To search for high-isospin candidates far from decay stability, as a reference, an empirical formula $Z=A /\left(1.98+0.0155 A^{2 / 3}\right)$ for the $\beta$-stability line is used here, which denotes the location of the maximum of the binding energy per nucleon or the minimum of the $Q$-value for $\beta$-decay [89, 90]. With this in mind, we take the neutron-deficient superheavy ${ }^{254} \mathrm{Rf}$ nucleus as an example to investigate the effects of the spin-orbit coupling strength and the nuclear surface diffuseness on potential energy curves with and without triaxiality, as shown in Fig. 8. The parameters $(a, \lambda)$ are slightly modified between a narrow domain on the basis of the initial values $(0.70,36)$, namely the universal values, according to the isospin-dependent function rela- 
tionship given in Ref. 87. Of course, the increased combination $(0.73,38)$ of the $(a, \lambda)$ parameters for protons is accordingly expected in this proton-rich nucleus. One can see from Fig. 8 that the fission barrier can be slightly raised, indicating that the corresponding fission probability will increase to some extent. A further test and improvement of model parameters, including the macroscopic part, will be done in our future work.

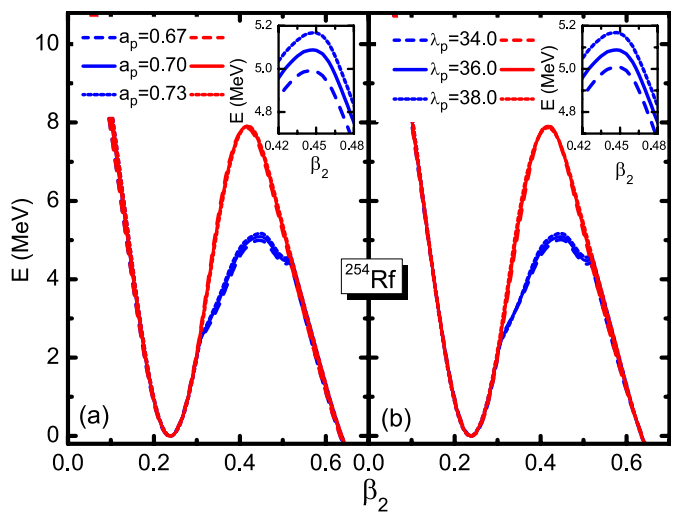

Fig. 8. Similar to Fig. 1(b), but the energy curves with adjusted surface diffuseness $a$ (a) and spinorbit strength $\lambda$ (b) for the selected neutrondeficient nucleus ${ }^{254} \mathrm{Rf}$. All other potential parameters are identical with those of universal values. The red (blue) lines denote the potential energy curves along the of axial (triaxial) fission path.

\section{Summary}

In summary, we have systematically presented the axial and triaxial fission barriers for 95 even-even transuranium nuclei ranging from ${ }^{228} \mathrm{Pu}$ to ${ }^{294} \mathrm{Og}$. The calculations were carried out using the pairing self-consistent potential energy surface approach with universal WoodsSaxon parameter set in multidimensional $\left(\beta_{2}, \gamma, \beta_{4}\right)$ de- formation space with the inclusion of triaxial shape degree of freedom. Our analysis shows that the main contribution of the fission barrier originates from the microscopic energy, especially the shell correction, though the pairing correction and macroscopic energy sometimes play a rather important role. Relative to the axially symmetric case, by allowing for triaxial deformation, the height and/or width of the inner fission barrier may be reduced considerably in most nuclei (e.g., more than 4 $\mathrm{MeV}$ in ${ }^{252} \mathrm{Cf}$ ), which will lead to a significant increase of the penetration probability in the spontaneous fission process. A systematic comparison of the present results with experimentally determined fission barriers shows a reasonable agreement (with rms deviation of $0.38 \mathrm{MeV}$ ) in the available actinide nuclei. Calculated energy curves with and without triaxial deformation in the actinide and superheavy regions indicate the influence of triaxiality on not only the heights but also the widths of the fission barriers with increasing nucleon number. Similar to mass calculations, it seems that to ensure the right fission barriers without unnecessary loss of CPU time, the selection of suitable deformation space is important, since missing any critical deformation degree of freedom may result in an overestimation of the calculated barriers. However, the underestimation of the theoretical calculations may be attributed to the choice of the potential parameters to some extent. For instance, the adjustment of the strength of spin-orbit coupling and the surface diffuseness will increase the height of the fission barrier. Therefore, besides the macroscopic energy, the perfect combination of the deformation space and potential parameter set will be a critical condition to guarantee good fission barriers in such phenomenological nuclear meanfield calculations. This systematic investigation should be helpful to test and develop the model in future as well as to understand and predict the fission properties in the even-even transuranium nuclei synthesized so far. A similar formulation for dynamic fission barriers is currently under way by including the Coriolis effect in the model Hamiltonian.

\section{References}

1 A. N. Andreyev, K. Nishio, and K-H Schmidt, Rep. Prog. Phys., 81: 016301 (2018)

2 R. Eichler, J. Phys.: Conf. Ser., 420: 012003 (2013)

3 S. Hofmann and G. Münzenberg, Rev. Mod. Phys., 72: 733 (2000)

4 Yu. Ts. Oganessian, A. Sobiczewski, and G. M. Ter-Akopian, Phys. Scr., 92: 023003 (2017)

5 F. P. Heßberger, Eur. Phys. J. A, 53: 75 (2017)

6 M. Arnould and K. Takahashi, Rep. Prog. Phys., 62: 395 (1999)

7 A. Mamdouh, J. M. Pearson, M. Rayet, and F. Tondeur, Nucl. Phys. A, 679: 337 (2001)
8 N. Bohr and J. A. Wheeler, Phys. Rev., 56: 426 (1939)

9 R. Capote, M. Herman, P. Obložinský, P. G. Young, S. Goriely, T. Belgya, A. V. Ignatyuk, A. J. Koning, S. Hilaire, V. A. Plujko, M. Avrigeanu, O. Bersillon, M. B. Chadwick, T. Fukahori, Z. Ge, Y. Han, S. Kailas, J. Kopecky, V. M. Maslov, G. Reffo, M. Sin, E. Sh. Soukhovitskii, and P. Talou, Nucl. Data Sheets, 110: 3107 (2009)

10 V. V. Pashkevich, Nucl. Phys. A, 133: 400 (1969)

11 P. Möller and S. G. Nilsson, Phys. Lett. B, 31: 283 (1970)

12 B. N. Lu, E. G. Zhao, and S. G. Zhou, Phys. Rev. C, 85: 011301(R) (2012)

13 B. N. Lu, J. Zhao, E. G. Zhao, and S. G. Zhou, Phys. Rev. C, 89: 014323 (2014)

14 P. Möller, A. J. Sierk, and A. Iwamoto, Phys. Rev. Lett, 92: 
$072501(2004)$

15 A. Sobiczewski and M. Kowal, Phys. Scr., T125: 68 (2006)

16 A. Dobrowolski, K. Pomorski, and J. Bartel, Phys. Rev. C, 75: 024613 (2007)

17 P. Möller, A. J. Sierk, T. Ichikawa, A. Iwamoto, R. Bengtsson, H. Uhrenholt, and S. Åberg, Phys. Rev. C, 79: 064304 (2009)

18 A. Dobrowolski, B. Nerlo-Pomorska, K. Pomorski, and J. Bartel, Acta Phys. Pol. B, 40: 705 (2009)

19 M. Kowal, P. Jachimowicz, and A. Sobiczewski, Phys. Rev. C, 82: 014303 (2010)

20 P. Möller, A. J. Sierk, T. Ichikawa, A. Iwamoto, and M. Mumpower, Phys. Rev. C, 91: 024310 (2015)

21 A. Staszczak, A. Baran, J. Dobaczewski, and W. Nazarewicz, Phys. Rev. C, 80: 014309 (2009)

22 M. Bender, K. Rutz, P.-G. Reinhard, J. A. Maruhn, and W. Greiner, Phys. Rev. C, 58: 2126 (1998)

23 L. Bonneau, P. Quentin, and D. Samsoen, Eur. Phys. J. A, 21: $391(2004)$

24 A. Staszczak, J. Dobaczewski, and W. Nazarewicz, Acta Phys. Pol. B, 38: 1589 (2007)

25 A. K. Dutta, J. M. Pearson, and F. Tondeur, Phys. Rev. C, 61: 054303 (2000)

26 H. Abusara, A. V. Afanasjev, and P. Ring, Phys. Rev. C, 82: 044303 (2010)

27 Z. P. Li, T. Nikšić, D. Vretenar, P. Ring, and J. Meng, Phys. Rev. C, 81: 064321 (2010)

28 P. Ring, H. Abusara, A. V. Afanasjev, G. A. Lalazissis, T. Nikšić, and D. Vretenar, Int. J. Mod. Phys. E, 20: 235 (2011)

29 H. Abusara, A. V. Afanasjev, and P. Ring, Phys. Rev. C, 85: $024314(2012)$

30 S. E. Agbemava, A. V. Afanasjev, D. Ray, and P. Ring, Phys. Rev. C, 95: 054324 (2017)

31 S. W. Ødegård, G. B. Hagemann, D. R. Jesen, M. Bergström, B. Herskind, G. Sletten, S. Törmänen, J. N. Wilson, P. O. Tjøm, I. Hamamoto, K. Spohr, H. Hübel, A. Görgen, G. Schönwasser, A. Bracco, S. Leoni, A. Maj, C. M. Petrache, P. Bednarczyk, and D. Curien, Phys. Rev. Lett, 86: 5866 (2001)

32 R. Bengtsson, H. Frisk, F. R. May, and J. A. Pinston, Nucl. Phys. A, 415: 189 (1984)

33 K. Starosta, T. Koike, C. J. Chiara, D. B. Fossan, D. R. LaFosse, A. A. Hecht, C. W. Beausang, M. A. Caprio, J. R. Cooper, R. Krücken, J. R. Novak, N. V. Zamfir, K. E. Zyromski, D. J. Hartley, D. L. Balabanski, J. Y. Zhang, S. Frauendorf, and V. I. Dimitrov, Phys. Rev. Lett, 86: 971 (2001)

34 H. L. Wang, J. Yang, M. L. Liu, and F. R. Xu, Phys. Rev. C, 92: 024303 (2015)

35 Q. Z. Chai, H. L. Wang, Q. Yang, and M. L. Liu, Chin. Phys. C, 39: 024101 (2015)

36 Q. Yang, H. L. Wang, M. L. Liu, and F. R. Xu, Phys. Rev. C, 94: 024310 (2016)

37 H. L. Wang, H. L. Liu, F. R. Xu, and C. F. Jiao, Chin. Sci. Bull., 57: 1761 (2012)

38 http://www.nndc.bnl.gov/

39 M. G. Itkis, Yu. Ts. Oganessian, and V. I. Zagrebaev, Phys. Rev. C, 65: 044602 (2002)

40 Yu. Ts. Oganessian, J. Phys. G: Nucl. Phys., 34: R165 (2007)

41 K. Rutz, M. Bender, T. Bürvenich, T. Schilling, P.-G. Reinhard, J. A. Maruhn, and W. Greiner, Phys. Rev. C, 56: 238 (1997)

42 B. Belgoumène, J. Dudek, and T. Werner, Phys. Lett. B, 267: 431 (1991)

43 J. Dudek, B. Szpak, M. G. Porquet, H. Molique, K. Rybak, and B. Fornal, J. Phys. G: Nucl. Phys., 37: 064301 (2010)

44 J. Dudek, B. Szpak, B. Fornal, and A. Dromard, Phys. Scr., T154: 014002 (2013)

45 F. R. Xu, P. M. Walker, J. A. Sheikh, and R. Wyss, Phys. Lett. B, 435: 257 (1998)

46 J. R. Nix, Annu. Rev. Nucl. Sci., 22: 65 (1972)
47 W. Nazarewicz, R. Wyss, and A. Johnson, Nucl. Phys. A, 503: $285(1989)$

48 V. M. Strutinsky, Nucl. Phys. A, 95: 420 (1967)

49 M. Brack, J. Damgaard, A. S. Jensen, H. C. Pauli, V. M. Strutinsky, and C. Y. Wong, Rev. Mod. Phys 44: 320 (1972)

50 P. H. Regan, F. R. Xu, P. M. Walker, M. Qi, A. K. Rath, and P. D. Stevenson, Phys. Rev. C, 65: 037302 (2002)

51 H. L. Liu, F. R. Xu, P. M. Walker, and C. A. Bertulani, Phys. Rev. C, 83: 011303 (2011)

52 W. Nazarewicz, J. Dudek, R. Bengtsson, T. Bengtsson, and I. Ragnarsson, Nucl. Phys. A, 435: 397 (1985)

53 A. Bohr, Mat. Fys. Medd. K. Dan. Vidensk. Selsk. 26: 1 (1952)

54 A. Bohr and B. R. Mottelson, Nuclear Structure, Vol. II (World Scientific, Singapore, 1998)

55 W. D. Myers and W. J. Swiatecki, Nucl. Phys., 81: 1 (1966)

56 P. Möller and J. R. Nix, At. Data and Nucl. Data Tables, 39: 213 (1988)

57 P. Möller, W. D. Myers, W. J. Swiatecki, and J. Treiner, At. Data and Nucl. Data Tables, 39: 225 (1988)

58 K. Pomorski and J. Dudek, Phys. Rev. C, 67: 044316 (2003)

59 S. Ćwiok, J. Dudek, W. Nazarewicz, J. Skalski, and T. Werner, Comp. Phys. Comm., 46: 379 (1987)

60 M. Bolsterli, E. O. Fiset, J. R. Nix, and J. L. Norton, Phys. Rev. C, 5: 1050 (1972)

61 R. Bengtsson, J. Dudek, W. Nazarewicz, and P. Olanders, Phys. Scr., 39: 196 (1989)

62 P. Möller, J. R. Nix, W. D. Myers, and W. J. Swiatecki, At. Data Nucl. Data Tables, 59: 185 (1995)

63 J. Dudek, W. Nazarewicz and T. Werner, Nucl. Phys. A, 341: $253(1980)$

64 G. A. Leander, J. Dudek, W. Nazarewicz, J. R. Nix, and Ph. Quentin, Phys. Rev. C, 30: 416 (1984)

65 J. Dudek and T. Werner, J. Phys. G: Nucl. Phys., 4: 1543 (1978)

66 J. Dudek, A. Majhofer, J. Skalski, T. Werner, S. Ćwjok and W. Nazarewicz, J. Phys. G: Nucl. Phys., 5: 1359 (1979)

67 J. Dudek, Z. Szymański and T. Werner, Phys. Rev. C, 23: 920 (1981)

68 R. Nojarov, J. Phys. G: Nucl. Phys., 10: 539 (1984)

69 H. C. Pradhan, Y. Nogami, and J. Law, Nucl. Phys. A, 201. 357 (1973)

70 P. Möller and J. R. Nix, Nucl. Phys. A, 536: 20 (1992)

71 G. Andersson, S. E. Larsson, G. Leander, P. Möller, S. G. Nilsson, I. Ragnarsson, S. Åberg, R. Bengtsson, J. Dudek, B. NerloPomorska, K. Pomorski, and Z. Szymański, Nucl. Phys. A, 268: 205 (1976)

72 K. Rutz, J. A. Maruhn, P.-G. Reinhard, and W. Greiner, Nucl. Phys. A, 590: 680 (1995)

73 S. Ćwiok, J. Dobaczewski, P.-H. Heenen, P. Magierski, and W. Nazarewicz, Nucl. Phys. A, 611: 211 (1996)

74 M. Samyn, S. Goriely, and J. M. Pearson, Phys. Rev. C, 72: $044316(2005)$

75 J.-P. Delaroche, M. Girod, H. Goutte, and J. Libert, Nucl. Phys. A, 771: 103 (2006)

76 G. N. Smirenkin, IAEA Report No. INDC(CCP)-359, Vienna, 1993

77 S. Karatzikos, A. V. Afanasjev, G. A. Lalazissis, and P. Ring, Phys. Lett. B, 689: 72 (2010)

78 J. Zhao, B. N. Lu, T. Nikšić, D. Vretenar, and S. G. Zhou, Phys. Rev. C, 93: 044315 (2016)

79 B. Pritychenko, M. Birch, B. Singh, and M. Horoi, At. Data Nucl. Data Tables, 107: 1 (2016)

80 A. Sobiczewski, I. Muntian, and Z. Patyk, Phys. Rev. C, 63: 034306 (2001)

81 P. Möller, A. J. Sierk, T. Ichikawa, and H. Sagawa, At. Data Nucl. Data Tables, 109-110: 1 (2016)

82 S. Goriely, F. Tondeur, and J. M. Pearson, At. Data Nucl. Data Tables, 77: 311 (2001) 
83 Y. Aboussir, J. M. Pearson, A. K. Dutta, and F. Tondeur, At. Data Nucl. Data Tables, 61: 127 (1995)

84 J. Dudek, W. Nazarewicz, and P. Olanders, Nucl. Phys. A, 420: 285 (1984)

85 K. Mazurek, J. Dudek, A. Maj, and D. Rouvel, Phys. Rev. C, 91: 034301 (2015)

86 J. Sadhukhan, W. Nazarewicz, and N. Schunck, Phys. Rev. C, 93: $011304(\mathrm{R})(2016)$
87 N. Wang, M. Liu, X. Z. Wu, and J. Meng, Phys. Lett. B, 734: $215(2014)$

88 Q. Zhi and Z. Ren, Phys. Lett. B, 638: 166 (2006)

89 P. Marmier and E. Sheldon, Physics of Nuclei and Particles (Academic Press, New York, 1971)

90 C. L. Wu, M. Guidry, and D. H. Feng, Phys. Lett. B, 387: 449 (1996) 